\title{
Epidemiology of trauma presentations to a major trauma centre in the North West of England during the COVID-19 level 4 lockdown
}

\author{
Kunal Rajput $^{1}\left[\right.$ Ajay Sud $^{1}\left[\right.$ [ $\cdot$ Michael Rees $^{1} \cdot$ Olga Rutka $^{1}$
}

Received: 29 June 2020 / Accepted: 18 September 2020 / Published online: 30 September 2020

๑) Springer-Verlag GmbH Germany, part of Springer Nature 2020

\begin{abstract}
Purpose The COVID-19 pandemic has impacted healthcare systems globally, little is known about the trauma patterns during a national lockdown. The aim of this study is to delineate the trauma patterns and outcomes at Aintree University Teaching Hospital level 1 Major Trauma Centre (MTC) during the COVID-19 lockdown imposed by the U.K. government. Methods A retrospective cohort study data from the Merseyside and Cheshire Trauma Audit and Research Network database were analysed. The 7-week 'lockdown period' was compared to a 7-week period prior to the lockdown and also to an equivalent 7 -week period corresponding to the previous year.

Results A total of 488 patients were included in the study. Overall, there was $37.6 \%$ and $30.0 \%$ reduction in the number of traumatic injuries during lockdown. Road traffic collisions (RTC) reduced by $42.6 \%$ and $46.6 \%$. RTC involving a car significantly reduced during lockdown, conversely, bike-related RTC significantly increased. No significant changes were noted in deliberate self-harm, trauma severity and crude mortality during lockdown. There was 1 mortality from COVID-19 infection in the lockdown cohort.

Conclusion Trauma continues during lockdown, our MTC has continued to provide a full service during lockdown. However, trauma patterns have changed and departments should adapt to balance these alongside the COVID-19 pandemic. As the U.K. starts its cautious transition out of lockdown, trauma services are required to be flexible during changes in national social restrictions and changing trauma patterns. COVID-19 and lockdown state were found to have no significant impact on survival outcomes for trauma.
\end{abstract}

Keywords COVID-19 $\cdot$ Lockdown $\cdot$ Pandemic $\cdot$ Trauma $\cdot$ Emergency surgery

\section{Introduction}

COVID-19 is caused by the severe acute respiratory syndrome coronavirus 2 (SARS-CoV-2) [1]. The British government's response to the pandemic included measures to reduce transmission and the reallocation of healthcare provisions to address the burden of the disease. This healthcare rearrangement had the potential to compromise trauma care. Guidelines have been published by the American College of Surgeons (ACS), the Royal College of Surgeons (RCS) and European Society of Trauma and Emergency Surgery (ESTES), collectively outlining the collateral impact COVID-19 potentiates to trauma services [1-3]. Trauma

Kunal Rajput

kunal.rajput@doctors.org.uk

1 Aintree University Hospital, Liverpool L9 7AL, UK services in the worst hit areas in northern Italy, during the pandemic, re-designed their practice to centralise trauma services, to enable local hospitals to focus on COVID-19 [4]. The expected mortality of up to $38 \%$ in surgical patients from the COVIDSurg collaborative gave further credence to the guidance to undertake, where possible, only immediately life-saving surgery [5].

Following the emergence of COVID-19 in the United Kingdom (U.K.), the government decided to implement a level 4 lockdown on 23 March 2020. The lockdown implied that all non-essential travels outside the individual's home were ceased, shopping for basic necessities was advised as infrequently as possible and one form of outside exercise a day, close to the residence, was permitted.

Across England and Wales, traumatic injuries are the leading cause of death in those under 40 years of age, with approximately 16,000 annual deaths [6]. Our institute, Aintree University Hospital is the regional Cheshire and 
Merseyside Major Trauma Center (MTC) for the northwest of England, with a dedicated trauma cover for adult traumatic injuries in a catchment area of 2.3 million residents. Evidence is scarce in relation to patterns and outcomes of trauma during this COVID-19 pandemic lockdown scenario.

Our aim is to quantify and qualify the trauma presentations at our MTC at the height of the COVID-19 pandemic level 4 lockdown. Furthermore, to delineate the incidence and outcomes of trauma patients during a lockdown state and provide recommendations for practice during a pandemic lockdown by outlaying our experience as an MTC in the northwest of England.

\section{Methods}

Data were retrospectively collected for the Trauma Audit and Research Network (TARN) database of all trauma team activations at our level 1 major trauma centre, Aintree University Hospital, Liverpool, England. Data were retrospectively analysed from the TARN database, divided into 7-week intervals. Datasets were divided into 3 cohorts with respect to time frame:

(1) Cohort "lockdown": Level 4 lockdown imposed by the

U.K. government (23 March 2020-10 May 2020)

(2) Cohort "pre-lockdown": 7 weeks prior to the imposed lockdown (27 January 2020-15 March 2020)

(3) Cohort "Year 2019": Corresponding 7 week equivalent period in the year 2019 (25 March 2019-12 May 2020).

A week prior to the lockdown (16 March 2020-22 March 2020) was excluded from this study, as the new concept of 'social distancing' was advised by the government during this week. This was to allow for community behavioral changes and varied levels of social distancing advice followed by the public.

Descriptive clinical and demographic information were included. The outcome measures were mortality rates, mechanism of injuries and severity of trauma according to the Trauma Injury Severity Score (ISS) [7] and deliberate selfharm (DSH). The ISS was divided categorically according to severity as $1-8,9-15$ and $16+$ as collected for the TARN database. Also, outcomes for major trauma from COVID-19 infection were explored.

Categorical data were represented as total counts and percentages. Significance of difference was statistically calculated using the chi-square test for categorical data. Confidence intervals were calculated using the 'modified Wald method'.

\section{Results}

121 traumatic injuries presented in the lockdown period, 173 presented during the pre-lockdown period and 194 presented in the corresponding year 2019 period. Comparatively, there was a reduction of $37.6 \%$ and $30.0 \%$ in the number of trauma presentations during lockdown period. The median age at presentation was 36 years (IQR 29-55) in the lockdown cohort, 47 years (IQR 30-64) in the pre-lockdown cohort and 41 years (IQR 27-62) in the year 2019 cohort. No significant differences were noted in the severity of trauma presentations. Mortality rates were reported at $7.4 \%$ during lockdown, when compared to $11 \%$ and $6.2 \%$ during the control cohorts (Table 1).

The most marked reduction occurred in road traffic collisions when compared to year 2019 (42.6\%) and reduction during pre-lockdown (46.6\%). A reduction was reported in
Table 1 Descriptive and trauma presentation characteristics

\begin{tabular}{lllll}
\hline Variables & $\begin{array}{l}\text { Year 2019 } \\
(\%)\end{array}$ & $\begin{array}{l}\text { Pre-lockdown } \\
(\%)\end{array}$ & $\begin{array}{l}\text { Lockdown } \\
(\%)\end{array}$ & $\begin{array}{l}\text { 95\% CI } \\
\text { Lockdown }\end{array}$ \\
\hline $\begin{array}{l}\text { Total number } \\
\text { Age, Median (IQR) }\end{array}$ & 194 & 173 & 121 & $\begin{array}{l}100.4,144.6 \\
\text { Gender }\end{array}$ \\
$\quad 41(27-62)$ & $47(30-64)$ & $36(29-55)$ & $25.2,49.8$ \\
Male & $141(74.4)$ & $113(65.3)$ & $95(78.5)$ & $85.1,102.8$ \\
Female & $49(25.6)$ & $59(34.7)$ & $25(21.5)$ & $17.4,34.8$ \\
ISS & & & & \\
$1-8$ & $115(59.5)$ & $98(56.6)$ & $60(50.0)$ & $49.4,70.6$ \\
$9-15$ & $32(16.4)$ & $29(16.8)$ & $35(29.0)$ & $26.1,45.5$ \\
$16+$ & $44(24.1)$ & $45(26.0)$ & $25(20.6)$ & $17.4,34.8$ \\
DSH & $20(10.3)$ & $24(13.9)$ & $14(11.6)$ & $8.3,22.5$ \\
Mortality rates & $6.2 \%$ & $11.0 \%$ & $9.9 \%$ & - \\
\hline
\end{tabular}

$N$ number, $C I$ confidence interval, IQR inter quartile range, ISS injury severity score, $D S H$ deliberate selfharm 
trauma presentations involving a car during the lockdown period (Table 2). In contrast, a significant increase was noted in trauma presentations from bike- or pushbike-related accidents during lockdown (7.4\%) when compared to pre-lockdown (3.5\%). Comparatively, the trend of traumatic injuries due to RTC (Fig. 1) reports an overall reduction in the lockdown cohort when compared to the other cohorts. The number of stabbings remained statistically stable at $17.4 \%$ in the lockdown cohort, $16.2 \%$ in the pre-lockdown cohort and $13.3 \%$ in the year 2019 cohort. The number of falls from more than $2 \mathrm{~m}$ height remained stable during the three-time periods (Table 2).

When reported as a weekly trend, the number of carrelated incidents during lockdown was highest at the beginning and end of lockdown (Fig. 2).

In the lockdown cohort, $62.5 \%$ of road traffic collisions involving a car were greater than 9 ISS. Conversely, they had a severity of greater than 9 ISS at $36.7 \%$ and $35.3 \%$ in the other cohorts.

There was an increase in the number of traumatic injuries at home during lockdown. In contrast, a reduction was noted in traumatic injuries in public (Table 3).

From the lockdown cohort, there were 4 positive swabs confirmed COVID-19 patients. From these, 1 patient died from the COVID-19 infection.

\section{Discussion}

The anticipation of the increased demand on trauma services during the 'first-wave' of the COVID-19 pandemic lockdown has not been borne out by our data. The public's adherence to lockdown principals is likely the primary contributing factor to the significant reduction in the number of
Table 2 Mechanisms of injury

Fig. 1 Total number of new traumatic presentations each week from RTC to our institute. $R T C$ road traffic collisions

\begin{tabular}{lcccc}
\hline Variables & $\begin{array}{l}\text { Year 2019 } \\
(\%)\end{array}$ & $\begin{array}{l}\text { Pre-lockdown } \\
(\%)\end{array}$ & $\begin{array}{l}\text { Lockdown } \\
(\%)\end{array}$ & 95\% CI lockdown \\
& $N=194$ & $N=173$ & $31(25.6)$ & $22.6,41.3$ \\
\hline RTC & $54(29.8)$ & $58(34.3)$ & $8(25.8)$ & $3.9,15.4$ \\
Car & $30(55.6)$ & $36(62.0)$ & $9(7.4)$ & $4.6,16.6$ \\
Bike/Pushbike & $7(3.6)$ & $6(3.5)$ & $10(32.3)$ & $5.3,17.8$ \\
Motorbike & $13(24.1)$ & $8(13.8)$ & $1(3.2)$ & $0,5.5$ \\
Others & $4(9.3)$ & $6(10.3)$ & $21(17.4)$ & $14.0,30.4$ \\
Stabbings & $25(13.3)$ & $28(16.2)$ & $26(21.5)$ & $18.2,35.9$ \\
Fall downstairs & $40(20.6)$ & $39(22.5)$ & $21(17.4)$ & $14.0,30.4$ \\
Fall $>2$ m & $24(12.4)$ & $23(13.3)$ & $10(8.3)$ & $5.3,17.8$ \\
Fall $<2$ m & $17(8.8)$ & $11(6.3)$ & $4(3.3)$ & $1.2,10.2$ \\
Blows & $9(4.6)$ & $2(1.2)$ & $2(1.6)$ & $0,7.5$ \\
Crush injuries & $4(2.1)$ & $1(0.6)$ & $6(5.0)$ & $2.5,12.9$ \\
Others & $12(6.4)$ & $7(2.9)$ & & \\
\hline
\end{tabular}

$C I$ confidence interval, $R T C$ road traffic collisions

\section{Weekly representation RTC}

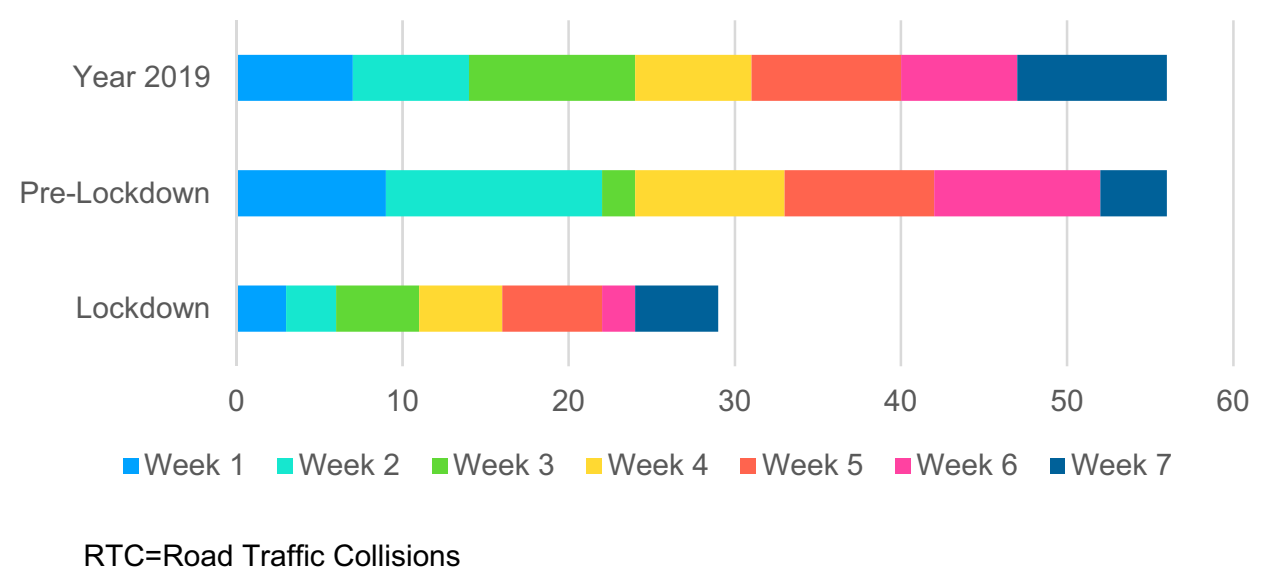


Fig. 2 Total number of new traumatic presentations each week from RTC involving a car to our institute. $R T C$ road traffic collisions

\section{Weekly representation of RTC involving a car}

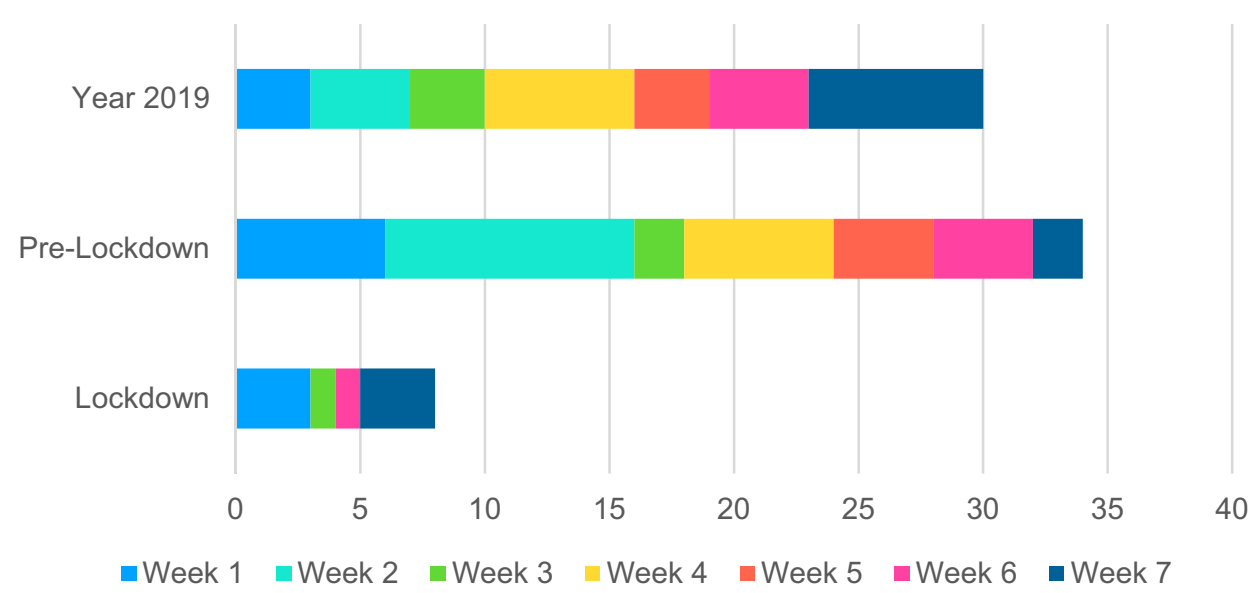

RTC=Road Traffic Collisions
Table 3 Place of injury

\begin{tabular}{lllll}
\hline Variables & $\begin{array}{l}\text { Year 2019 } \\
(\%) \\
N=194\end{array}$ & $\begin{array}{l}\text { Pre-lockdown } \\
(\%) \\
N=173\end{array}$ & $\begin{array}{l}\text { Lockdown } \\
(\%) \\
N=121\end{array}$ & $\begin{array}{l}\text { 95\% CI } \\
\text { lockdown }\end{array}$ \\
\hline Home & $69(35.6)$ & $71(41.0)$ & $64(52.9)$ & $53.3,74.5$ \\
Road & $50(25.8)$ & $58(33.5)$ & $35(28.1)$ & $26.1,45.5$ \\
Public & $70(36.1)$ & $40(23.1)$ & $22(18.2)$ & $14.8,31.5$ \\
Others & $4(2.1)$ & $1(0.6)$ & 0 & $0,4.5$ \\
\hline
\end{tabular}

$N$ number, CI confidence interval

major traumatic presentations during lockdown. Moreover, the wide-spread anxiety amongst the general population about contracting COVID-19 has likely reduced their traumagenic behavior. The perception by patients about potentially contracting COVID-19 in hospital was seen in all areas of emergency healthcare, with a 57\% reduction in accident and emergency admissions reported by 14 May 2020 when compared to the year 2019 [8].

The advent of social isolation has the prospect to break down social support networks and have an impact on mental health. Likewise, a report published by Garude et al. states an increase in hand-related traumas from DSH [9]. In contrast, our institution has noted an overall decrease in the numbers of serious traumatic injuries from DSH (including violent suicide and parasuicide attempts). This indicates a reluctance from individuals to self-inflict serious traumatic injuries, such as stabbings, fall from high structures and hanging during lockdown. This contradicts the experience seen in Hong Kong during the SARS outbreak and the institution of level 4 lockdown where the suicide rate was historically high, particularly in older populations, who were potentially socially more isolated
[10]. Unreported, non-presenting self-harm and cases not reaching the threshold for self-harm, have the potential to increase during lockdown and pandemic states. However, we have not extended this study to assess this. A potential explanation for a reduction in violent DSH is increase in non-violent modus operandi (such as overdoses). Substance misuse is a known risk factor for precipitating $\mathrm{DSH}$, as well as playing a role in mental health issues and self-harm. It would be interesting to focus on DSH during COVID-19 lockdown as a whole. The complex interaction between socio-economic, psychosocial, mental and physical well-being during COVID-19 lockdown will be studied extensively in the coming years [11].

In our study, RTC nearly halved in number. Data from the British department of transport show a 73\% drop in the number of motorists at the end of March 2020 [12]. With the majority of people working from home and with only essential travel being allowed, there were fewer vehicles on road. The number of presentations should be expected at near zero, as only essential travel was advised by the government. Christey et al. from New Zealand report the propensity for road traffic collisions to continue during lockdown [13]. When studying the trend of RTC involving a car, the highest numbers were noted at the start and end of lockdown. In week 1 , this can be attributed to the time taken for behavioral shifts in the community transitioning into lockdown. Likewise in week 6 , the increased likelihood of non-adherence to lockdown rules was experienced as rumours of easing lockdown restrictions emerged.

The department of transport has also reported an increase in the number of speeding motorists [14], potentially explaining the prevalence of car-related incidents during lockdown. As a result, serious traumatic presentation ( $>9$ ISS score) during the lockdown was more common. 
There was an increased incidence of bike- or pushbikerelated RTC during lockdown. This is likely due to the fact that riding a bike became the chosen mode of exercise for many people in warmer weather while keeping to the statutory $2 \mathrm{~m}$ distance. Moreover, bike retailers have reported an increase in bike sales during the COVID-19 lockdown [15], indicating an increase in novice bike riders with minimal riding experience.

The incidence of trauma at home significantly increased during lockdown ( $p$ value 0.002 and 0.04 ). Although the total number of traumatic events fell during lockdown, the number of falls from over $2 \mathrm{~m}$ height remained prevalent. These resulted from a tendency of people to perform DIY activities at home during lockdown, especially with the advent of spring. A significant increase in hand injuries associated with using tools at home while performing DIY activities during lockdown have been noted in other studies [9]. An increase was also noted in the proportion of stabbings encountered during the COVID-19 lockdown compared to other mechanisms of injury. These stabbings mostly occurred at home and involved individuals confined to the lockdown rules. Similar patterns have been reported with regard to increased violence at home during lockdown in the U.K.[16, 17].

In our study, no significant differences in mortality rates were reported. Moreover, the severity was unchanged, likely contributing to the stability in mortality rates. Our regional trauma centre emulated the guidance set by ESTES, limiting the number of health care staff present at the trauma call. This was achieved by a rapid primary survey, by an Accident and Emergency doctor, to assess the severity of the trauma while specialties were on standby to attend at short notice. This was done to prevent exposure to healthcare staff to any potential COVID-19 infection. Interestingly, this re-modelling of the trauma call has incurred no significant impact on the crude mortality during the lockdown.

Recommendations from ESTES, RCS and ACS report the necessity of full personal protective equipment (PPE) for all trauma teams when attending to a major trauma [1-3]. Moreover, Samsami et al. outline the incidence of asymptomatic COVID-19 infection in major trauma patients [18]. From our study, it is evident that trauma presentations rarely co-exist with concurrent COVID-19 infection, but the potential still exists. Importantly, it is difficult to assess the impact of COVID-19 on survival outcomes from major trauma.

The limitations of this study is the short study period, single-centre experience, only the inclusion of TARN Major trauma patients and the availability of comprehensive outcome data for all the patients included in the study. A larger, multi-centred study encompassing trauma patterns during lockdown will provide strength to the study. Moreover, the ISS has its limitations in defining nuances between exact severities of traumatic injuries, though ISS is the generally used as a prediction of survival. The inevitable next stage of this study would be to include directly comparable TARN data from previous years, and also to investigate in more detail the outcomes in major trauma patients infected with COVID-19.

\section{Conclusion}

Major Trauma presentations are a microcosm of the state of societal extremes. Though there was a reduction in road traffic collision-related major trauma admissions, those which occurred had more severe injuries, resulting in other trauma aetiologies making a greater proportion of presentations. Major Trauma and COVID-19 do not commonly co-exist as a discrete entity. The mobilisation of the general population as lockdown measures is relaxed potentiates an increase in traumatogenic behavior and a spike in the number of trauma presentations. Maintenance of an adaptive efficacious full capability Major Trauma Service is imperative in spite of the COVID-19 lockdown.

Data availability The data used to support the findings of this study are restricted by the TARN database. Data is available from TARN for access to confidential data. Requests for access to these data should be made to TARN.

\section{Compliance with ethical standards}

Conflict of interest The authors of this article (Kunal Rajput, Ajay Sud, Michael Rees and Olga Rutka) do not have any conflict of interest nor received any funding for this work.

\section{References}

1. Maintaining trauma center access and care during the COVID-19 pandemic: guidance document for trauma medical directors. In: American college of surgeons. 2020. https://www.facs.org/quali ty-programs/trauma/maintaining-access. Accessed 29 June 2020.

2. Coimbra R, Edwards S, Kurihara H, Bass GA, Balogh ZJ, Tilsed J, et al. European society of trauma and emergency surgery (ESTES) recommendations for trauma and emergency surgery preparation during times of COVID-19 infection. Eur J Trauma Emerg Surg. 2020. https://doi.org/10.1007/s00068-020-01364-7.

3. Intercollegiate general surgery guidance on COVID-19 UPDATE. In: the royal college of surgeons of Edinburgh. 2020; https://www. rcsed.ac.uk/news-public-affairs/news/2020/march/intercollegiate -general-surgery-guidance-on-covid-19-update. Accessed 26 June 2020.

4. Di Martino A, Faldini C. Trauma service reorganization in Bologna (Italy) during COVID-19 pandemic. Else Pub Health Emerg Collect. 2020. https://doi.org/10.1016/j.injury.2020.04.033.

5. COVIDSurg Collaborative. Mortality and pulmonary complications in patients undergoing surgery with perioperative SARSCoV-2 infection: an international cohort study. Lancet. 2020. https ://doi.org/10.1016/S0140-6736(20)31182-X(published online 
ahead of print, 2020 May 29, [published correction appears in Lancet. 2020 Jun 9]. S0140-6736(20)31182-X).

6. The Trauma audit and research network website. 2019. https:// www.tarn.ac.uk/Content.aspx? $\mathrm{ca}=2 \& \mathrm{c}=3860$. Accessed 6 June 2020.

7. Boyd CR, Tolson MA, Copes WS. Evaluating trauma care: the TRISS method. Trauma score and the injury severity score. J Trauma. 1987;27(4):370-8.

8. Evison C. A and E attendances and emergency admissions march 2020 statistical commentary. NHS England and NHS improvement. 2020. https://www.england.nhs.uk/statistics/wp-content/ uploads/sites/2/2020/04/Statistical-commentary-March-2020jf8hj.pdf. Accessed 6 June 2020.

9. Garude $\mathrm{K}$, et al. Patterns of adult and paediatric hand trauma during the COVID-19 lockdown. J Plast Reconstr Aesthet Surg. 2020. https://doi.org/10.1016/j.bjps.2020.05.087.

10. Cheung YT, Chau PH, Yip PS. A revisit on older adults suicides and Severe Acute respiratory syndrome (SARS) epidemic in Hong Kong. Int J Geriatr Psychiatry. 2008;23(12):1231-8. https://doi. org/10.1002/gps.2056.

11. Barber SJ, Kim H. COVID-19 worries and behavior changes in older and younger men and women. J Gerontol B Psychol Sci Soc Sci. 2020. https://doi.org/10.1093/geronb/gbaa0 68(gbaa068,published online ahead of print, 2020 May 19).

12. Transport use during the coronavirus (COVID-19) pandemic. In: driving and transport in the UK during coronavirus. 2020. https:// www.gov.uk/government/statistics/transport-use-during-the-coron avirus-covid-19-pandemic. Accessed 6 June 2020

13. Christey G, Amey J, Campbell A, Smith A. Variation in volumes and characteristics of trauma patients admitted to a level one trauma centre during national level 4 lockdown for COVID-19 in New Zealand. New Engl Med J. 2020;133:81-8.

14. Data about passengers, travelling to work, casualty rates and employment occupations, produced by Department for Transport. In: Driving and road transport. 2020. https://www.gov.uk/ government/statistical-data-sets/tsgb01-modal-comparisons\#table s-tsgb0117-to-tsgb0125. Accessed 6 June 2020.

15. Huge demand for bikes during lockdown set to grow further. In: the guardian. https://www.theguardian.com/business/2020/ may/11/huge-demand-for-cycles-during-lockdown-set-to-risefurther. Accessed 6 June 2020.

16. Hatchimonji JS, Swendiman RA, Seamon MJ, Nance ML. Trauma does not quarantine: violence during the Covid-19 pandemic. Ann Surg. 2020. https://doi.org/10.1097/SLA.0000000000003996.

17. Campbell AM, et al. An increasing risk of family violence during the Covid-19 pandemic: strengthening community collaborations to save lives. FSI Reports. 2020;2:100089. https://doi. org/10.1016/j.fsir.2020.100089.

18. Samsami M, Zebarjadi Bagherpour J, Nematihonar B, Tahmasbi H. COVID-19 pneumonia in asymptomatic trauma patients; report of 8 cases. Arch Acad Emerg Med. 2020;8(1):e46. 